\title{
Nouveaux procédés physiques d'exploration en biologie et en médecine
}

De récents développements dans l'instrumentation physique ont fourni aux biologistes de nouveaux et précieux outils pour analyser les structures moléculaires : la RMN à haut champ, en trois ou quatre dimensions, avec marquage isotopique, permettra bientôt l'analyse de protéines d'un poids moléculaire atteignant 30000 à 40000 . Le microscope à effet tunnel permet de visualiser les molécules avec une très grande résolution. De nouvelles sources de rayons $X$, fournies par des synchrotrons, de haute énergie et polychromatiques, facilitent enfin les études cristallographiques et pourraient avoir d'intéressantes applications en imagerie médicale.

\section{Bernard Jacrot}

\section{ADRESSE}

B. Jacrot : direcleur de recherches. Institut Max von Laue-Paul Langevin, avenue des
Martyrs, 156X, 38042 Grenoble Cedex, 'utilisation des méthodes physiques en biologie et en médecine n'est pas un phénomène nouveau. La découverte des cellules a résulté de l'utilisation du tout nouvel instrument qu'était alors le microscope. La découverte des rayons $\mathrm{X}$ a très rapidement permis la mise en œuvre de la première méthode d'imagerie médicale. Au cours du $\mathrm{XX}^{\mathrm{e}}$ siècle, les progrès de la physique ont largement contribué à ceux de la biologie. La détermination de la structure spatiale des protéines a été le résultat de la découverte, au début du siècle, de la diffraction par von Laue, de l'amélioration des sources de rayons $\mathrm{X}$ et de leurs détecteurs et enfin de l'invention des ordinateurs. Dans le domaine médical, la maîtrise des ultrasons a permis la mise au point d'une méthode d'imagerie médicale. La microscopie électronique joue un rôle essentiel en biologie et contribue à de nombreux diagnostics. Ce ne sont là que quelques exemples de l'impact des méthodes physiques en biologie et en médecine. Ces applications sont si complètement intégrées et assimilées par les chercheurs ou les médecins qu'ils en oublient qu'il s'agit de méthodes physiques. Les applications plus récentes sont évidemment moins bien assimilées et le but de cet article est de passer en revue ce que certains progrès récents en physique et en instrumentation ont apporté et pourront apporter en biologie et en médecine.

Cette revue ne prétend pas être $\mathrm{m} / \mathrm{s} n^{\circ} 4$, vol. 7, avril 91 
exhaustive ni totalement objective dans le choix des sujets traités. Il ne sera question que de ce qui permet de " voir " la matière. En médecine, il s'agit d'imagerie ; en biologie, cela doit s'entendre au sens de "voir " les atomes d'une macromolécule ou d'un assemblage de macromolécules. On parlera surtout des possibilités offertes par les sources de rayons X, en particulier les sources très intenses. C'est un sujet qui n'a jamais été abordé dans ce journal. Auparavant, les perspectives ouvertes en biologie par les progrès en RMN (résonance magnétique nucléaire) et par la microscopie à effet tunnel seront brièvement exposées. On ne traitera pas ici des développements considérables et d'une grande importance pratique qui sont apparus récemment dans les techniques d'imagerie médicale telles que l'imagerie ultrasonore, la tomographie par émission de positons ou la scintigraphie.

\section{La microscopie à effet tunnel}

Inventée en 1982 par Binning et Rohrer, qui reçurent pour cela le prix Nobel dès 1986, décrite dans médecine/sciences la même année $(\mathrm{m} / \mathrm{s}$ $n^{\circ} 7$, vol. 2, p. 104), la méthode consiste à mesurer le très faible courant qui s'établit entre un objet conducteur et une pointe métallique quand cette pointe est suffisamment proche de l'objet. Comme l'intensité de ce courant varie très rapidement avec la distance entre la pointe et l'échantillon, on peut étudier les surfaces des solides avec une résolution qui peut atteindre - dans les meilleures conditions $-0,1 \mathrm{~nm}$, permettant alors de voir les atomes. Cette microscopie nouvelle est l'objet de l'attention de beaucoup de chercheurs qui espèrent qu'elle offrira un jour la possibilité de voir les atomes d'une molécule biologique. Cela n'est pas possible en microscopie électronique, en tous cas avec des molécules isolées, à cause des effets destructeurs du faisceau d'électrons. Ces effets sont absents en microscopie à effet tunnel, d'où les grands espoirs mis dans la méthode. On peut alors rêver et, pourquoi pas, espérer séquencer l'ADN par cette méthode $\left(\mathrm{m} / \mathrm{s} n^{\circ} 7\right.$, vol. 4, p. 451, $n^{\circ} 10$, vol. 6, p. 1007 $\mathrm{m} / \mathrm{s} n^{\circ} 4$, vol. 7, avril 91

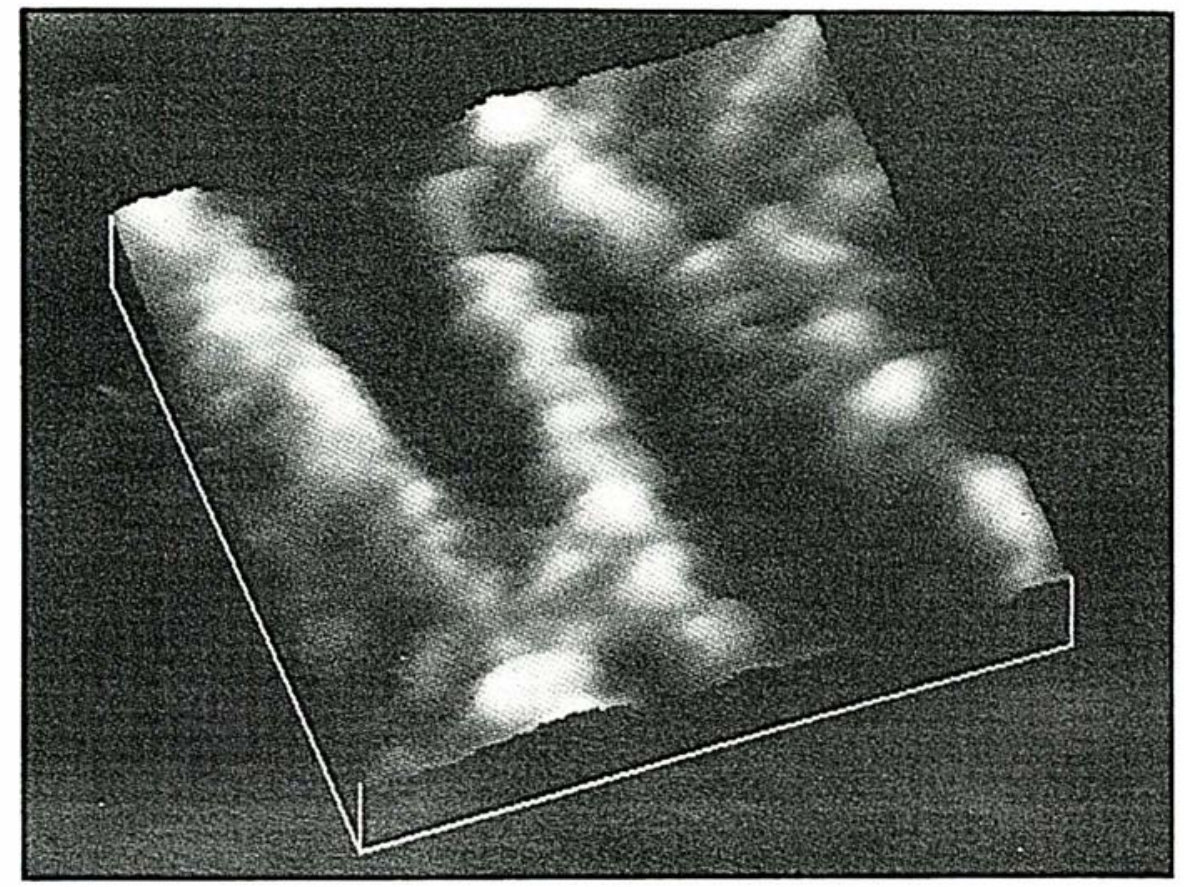

Figure 1. Filaments d'actine observés avec un microscope à force atomique. L'échantillon a été obtenu en laissant polymériser l'actine $G$ sur une surface de mica. L'image a $61 \mathrm{~nm}$ de côté $/$ Weisenhorn AL, Drake B, Prater $C B$, et al. Immobilized proteins in buffer imaged at molecular resolution by atomic force microscopy. Biophys J $1990 ; 58$ : 1251-8). Cette image donne une bonne idée de ce que l'on parvient actuellement à obtenir à présent avec un échantillon en milieu aqueux. La résolution est encore loin de permettre de voir les atomes comme cela est déjà possible avec des échantillons métalliques.

et [1]). Que peut-on vraiment espérer?

Les progrès sont certains. On sait maintenant observer des échantillons sous eau (ce qui paraît essentiel pour des molécules biologiques) en gardant, au moins dans le cas des échantillons métalliques, la résolution atomique. De beaux travaux ont été réalisés en physique qui ont permis de suivre l'" électrodéposition " d'une couche de plomb sur une surface d'or. Mais, avec les molécules biologiques, on est encore loin de pouvoir faire des travaux équivalents. Dans son principe, la méthode ne permet de visualiser que des matériaux conducteurs qui permettent à un courant électrique de circuler entre l'échantillon et la pointe de la sonde; les premières images de molécules bio- logiques ont été obtenues avec des échantillons recouverts d'une mince couche métallique. La méthode perd alors beaucoup de son intérêt. Plus récemment [2], on a pu voir des molécules nues d'ADN. Le mécanisme qui permet à un courant de s'établir n'est, dans ce cas-là, pas encore bien compris. Il est légitime d'espérer des progrès dans la compréhension de ce mécanisme. Des progrès sont également nécessaires dans l'amélioration de la résolution. Avec les molécules biologiques, cette résolution n'est pour le moment, le plus souvent, pas meilleure que celle obtenue en microscopie électronique. On est encore loin de voir les atomes. Une image, récemment publiée [3], d'ADN dans l'ultra-vide est cependant encourageante. 
Le microscope à force atomique est une variante du microscope à effet tunnel dans laquelle on étudie une surface de la même façon que l'on lit un disque en vinyle. La mécanique est simplement plus fine pour permettre de lire avec une précision meilleure que le nanomètre. L'échantillon n'a pas besoin d'être conducteur. Un des premiers objets biologiques observés a été du fibrinogène dont on a pu suivre la coagulation en temps réel [4]. C'est une expérience très prometteuse, sans doute la plus convaincante de toutes celles réalisées avec des échantillons biologiques. Il n'est évidemment pas possible de suivre une réaction en temps réel en microscopie électronique puisque, avec cette méthode, l'échantillon est soit déshydraté, soit enrobé dans la glace. En microscopie optique, même avec ses récents développements tels que le microscope confocal, la résolution est très insuffisante pour voir une protéine polymériser. Les microscopes à effet tunnel ou à force atomique sont donc, à ce jour, les seuls instruments permettant de visualiser de tels phénomènes (figure 1, p. 327). Une difficulté commune à ces deux méthodes demeure le repérage de l'échantillon, car le champ d'observation est très limité. Un couplage avec un microscope électronique sera peutêtre une façon de résoudre ce problème.

\section{La résonance magnétique nucléaire (RMN)}

3. Driscoll RJ, Youngquist MG, Baldeschwieler JD. Atomic scale imaging of DNA using scanning tunnelling microscopy. Nature $1990 ; 346: 294-6$.

4. Drake B, Prater, CB, Weisenhorn AL, et al. Imaging crystals, polymers, and processes in water with the atomic force microscope. Science 1989 ; 243: 1586-9.

5. Kline AD, Braun W, Wütrich K. Studies by $1 \mathrm{H}$ nuclear magnetic resonance and distance geometry of the solution conformation of the $\alpha$-amylase inhibitor tendamistat. J Mol Biol 1986 ; 189 : 377-82.

6. Boulanger Y, Vinay P. Étude des ćlectrolytes intracellulaires par rćsonance magnćtique nucléaire. médecine/sciences 1987 ; $3: 288-92$.

7. Kay LE, Clore GM, Bax AD, Gronenborn A. Four-dimensional heteronuclear triple-resonance NMR spectroscopy of interleukin-1 $\beta$ in solution. Science 1990 . 249: 411-4.
Cette méthodologie utilise la mesure mánportement du moment champ magnétique intense. Ce champ magnétique oscillant à fréquence (fréquence de résonanc noyau. Les possibilités de cette méthode croissent avec l'intensité des champs magnétiques que l'on sait produire. L'une de ses applicatior est une méthode d'imagerie médicale qui est maintenant d'usage courant

La RMN permet également des études de physiologie cellulaire [5]. Un exemple en a été donné dans ce journal [6]. Cette méthode non invasive a des applications nombreuses tant dans les hôpitaux. en physiologie animale qu'en physiologie végétale.

Plus récente est l'utilisation de la RMN pour la détermination, à l'échelle atomique, de la structure de macromolécules. Depuis plusieurs décennies, la RMN est utilisée par les chimistes pour étudier les structures et les mouvements. Tout repose sur le fait que la fréquence de résonance d'un noyau $\left({ }^{1} \mathrm{H},{ }^{15} \mathrm{~N},{ }^{13} \mathrm{C}\right.$, ${ }^{31} \mathrm{P}$ ) dépend de son environnement électronique (déplacement chimique). L'interprétation de ces déplacements de fréquence, qui est possible pour une petite molécule, était considérée jusqu'à il y a une dizaine d'années comme impossible pour une protéine. Ce qui a permis de progresser a été la mise au point d'une méthodologie (RMN à deux dimensions) qui consiste à déterminer quels sont les noyaux qui sont couplés, en cherchant quelles sont les paires dans lesquelles l'excitation de l'un des noyaux perturbe le signal de résonance de l'autre. Ces couplages sont de deux sortes : ceux s'effectuant au travers de liaisons covalentes et ceux s'effectuant dans l'espace. Ce n'est pas le lieu ici de dire comment se font les mesures. Retenons que, en première approximation, le premier type de couplage permet d'attribuer les fréquences de résonance au bon noyau en suivant la chaîne polypeptidique. Le deuxième permet de dire que, si deux noyaux (en général des noyaux d'hydrogène, c'est-à-dire des protons) sont couplés, leur distance est inférieure à environ $0,5 \mathrm{~nm}$. Cette dernière information est utilisable directement pour déterminer la proximité d'acides aminés dans la chaîne polypeptidique repliée. On peut ainsi déterminer tout un ensemble de proximités. Tout cela a été long à mettre au point et des progrès sont encore attendus.

La première protéine dont la structure ait été ainsi établie, en l'absence de toute information cristallographique, fut un inhibiteur de l'amylase, long de 75 acides aminés [5]. Le repliement trouvé pour la chaîne polypeptidique a été confirmé, a posteriori, par la détermination de la structure par les méthodes classiques de cristallographie aux rayons $\mathrm{X}$; (figure 2, p. 329). La méthode était donc validée. Son intérêt principal est 


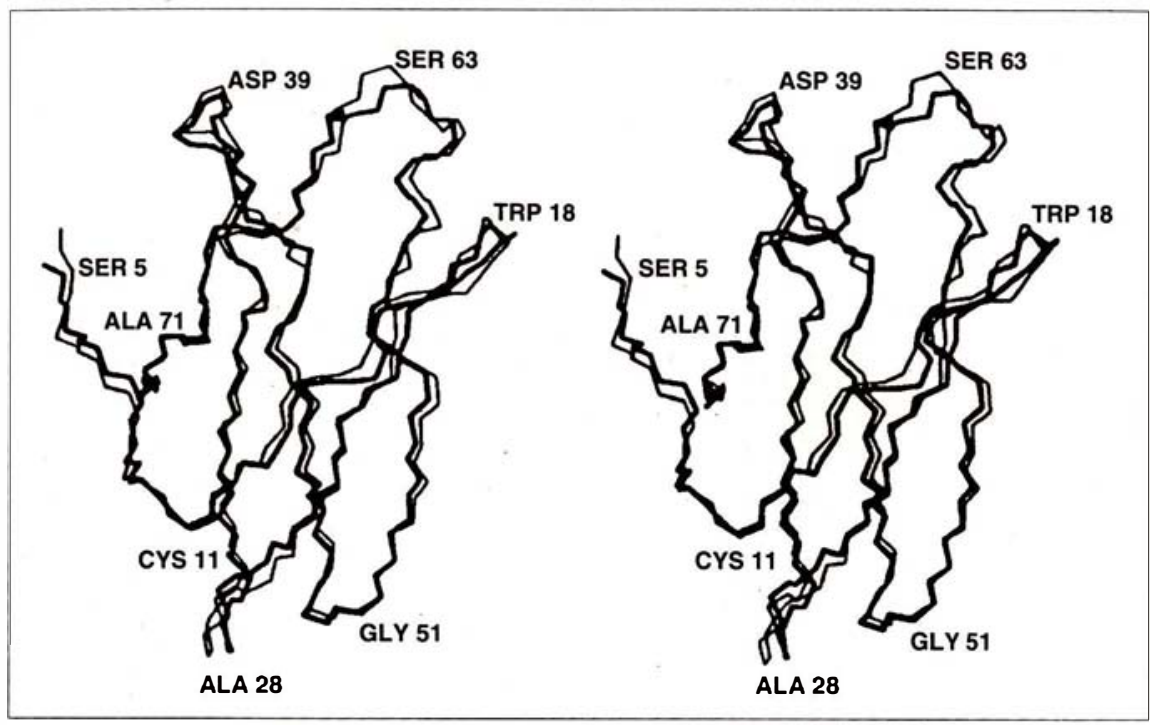

Figure 2. Comparaison du repliement de la chaîne polypeptidique déterminé par la diffraction des rayons $X$ (courbe en trait fin) ou par la $R M N$ (courbe en trait épais). La protéine est un inhibiteur de l'amylase qui est constitué de 75 acides aminés. Les deux images permettent d'avoir une vision stéréoscopique. La position, le long de la chaîne, de quelques acides aminés, repérés par leur position dans la séquence, aide à suivre cette chaîne. Les deux déterminations furent faites indépendamment l'une de l'autre. Les petites différences résultent en partie de la précision de ces déterminations et en partie de légères différences dans les parties les plus externes, suivant que les molécules sont en solution ou ordonnées à l'intérieur d'un cristal. ( $D^{\prime}$ 'après Billeter M, Kline AD, Braun W, Huber R, Wuthrich K. J Mol Biol 1989 ; 206 : 677-87.)

qu'il n'est pas nécessaire d'utiliser des monocristaux, toujours difficiles à obtenir. Elle est, en revanche, limitée à de petites protéines qui permettent une distinction des fréquences de résonance des différents noyaux. Dans les premières applications, la méthode n'était applicable qu'à des protéines de poids moléculaire inférieur à 10000 . L'utilisation de champs magnétiques très intenses permet d'augmenter la résolution. On utilise maintenant couramment des champs de 14 teslas (fréquence de résonance du proton à $600 \mathrm{MHz}$ ). Un appareil commercial est annoncé qui utilisera un champ de 17,5 teslas (1 tesla $=10000$ Gauss). Il existe, en France, un projet de spectromètre RMN utilisant un champ de 20 teslas. La deutérisation de certains acides aminés (qui conduit au remplacement de protons par des deutérons, qui ont un moment magnétique différent et donc des fréquences de résonance différentes) permet de réduire le nombre de signaux et de $m / s$ n 4, vol. 7, avril 91 lite l'interprétation; il jouera dans l'avenir un rôle clé. La combinaison de la $\mathrm{RMN}$, de la cristallographie et de la modélisation moléculairc permettra de spectaculaires progrès dans la connaissance des structures, en particulier des modifications locales résultant de la substitution d'un acide aminé par un autre.

\section{Diffraction \\ des rayons $X$ et détermination des structures}

Au risque de dirc des choses connucs de tous les lecteurs, je rappellerai d'abord qu'il y a deux façons d'utiliser les rayons $\mathrm{X}$ pour étudier la matière. Dans la première, on utilise le fait que lorsqu'un faisceau de rayons $\mathrm{X}$ traverse la matière, une partie est déviée ou absorbée. La mesure du faisceau transmis donne une information sur les matériaux au travers desquels il est passé. Cela est la base de l'imagerie par rayons $\mathrm{X}$ utilisée en médecine et dans de nombreuses applications industriclles depuis plus de soixante-dix ans. Dans la deuxième méthode, on utilisc les faisceaux déviés (on dit diffusés) par les atomes de l'échantillon que l'on veut analyser, pour déduire par le calcul l'organisation de ces atomes. Cette méthode, ditc diffraction des rayons $\mathrm{X}$, est utilisée, depuis les travaux de von Laue au début du siècle, pour déterminer la structure des molécules. Son utilité en biologie a été démontrée par les travaux de Max Perutz et John Kendrew qui déterminèrent, il y a un peu plus de trente ans, la structure de la myoglobine et celle de l'hémoglobinc. Depuis, elle est utilisée par des dizaines de laboratoires et elle a permis de déterminer la structure de centaines d'enzymes, d'acides nucléiques, de virus et d'autres assemblages macromoléculaires.

Avec un microscope (optique ou électronique), on observe un objet par l'intermédiaire des ondes (photons ou électrons) diffusées par les atomes de l'objet. Ces ondes passent au travers d'un système optique et une image est obtenue. Avec un microscope optique, la résolution est limitée par la longueur d'onde de la lumière, qui est tellement grande par rapport aux 
distances interatomiques que les contributions d'atomes voisins sont indiscernables. Avec le microscope électronique, la longueur d'onde des électrons est petite par rapport à la distance entre atomes et l'on peut voir les atomes dans un échantillon métallique. Dans l'observation d'objets biologiques, la limitation en résolution est la conséquence de la destruction partielle de l'échantillon par le faisceau d'électrons.

Avec les rayons $\mathrm{X}$, on utilise une longueur d'onde de l'ordre de $0,1 \mathrm{~nm}$ et les sources sont suffisamment peu intenses pour que l'échantillon ne soit pas complètement détruit par les effets du rayonnement. Malheureusement, on ne sait pas construire de lentilles pour les rayons $X$. Cela est lié au fait que la matière $a$ un indice de réfraction très voisin de 1 pour ce rayonnement et que ce dernier n'a pas de charge électrique. Il faut donc travailler directement avec les rayons $\mathrm{X}$ diffusés par l'objet observé, une macromolécule ou un assemblage tel qu'un virus, par exemple. Il est à noter que les projets de "microscopes à rayons $\mathrm{X}$ " dont il est parfois fait état reposent sur l'utilisation des rayons $\mathrm{X}$ " mous ", dont la longueur d'onde, de l'ordre de $10 \mathrm{~nm}$, ne permet pas d'atteindre une résolution atomique.

Avec un objet unique, une seule protéine par exemple, le signal est beaucoup trop faible pour que quoi que ce soit puisse être mesuré. En effet, les faisceaux de rayons $\mathrm{X}$ sont beaucoup moins intenses que ceux d'électrons dans un microscope, et leur interaction avec la matière est plus faible. Pour prendre l'image d'une molécule en microscopie électronique, on utilise entre 100 et 10000 électrons par $\mathrm{nm}^{2}$. Avec la plus faible de ces doses, l'image de la molécule est pratiquement invisible à l'œil nu sur le cliché. Les sources les plus intenses de rayons $\mathrm{X}$ disponibles dans les laboratoires permettent d'envoyer $10^{-4}$ photon par seconde sur la même surface. C'est pourquoi les problèmes de destruction de l'échantillon se posent de façon complètement différente. Dans un microscope électronique, l'échantillon est perturbé, au point que la résolution est

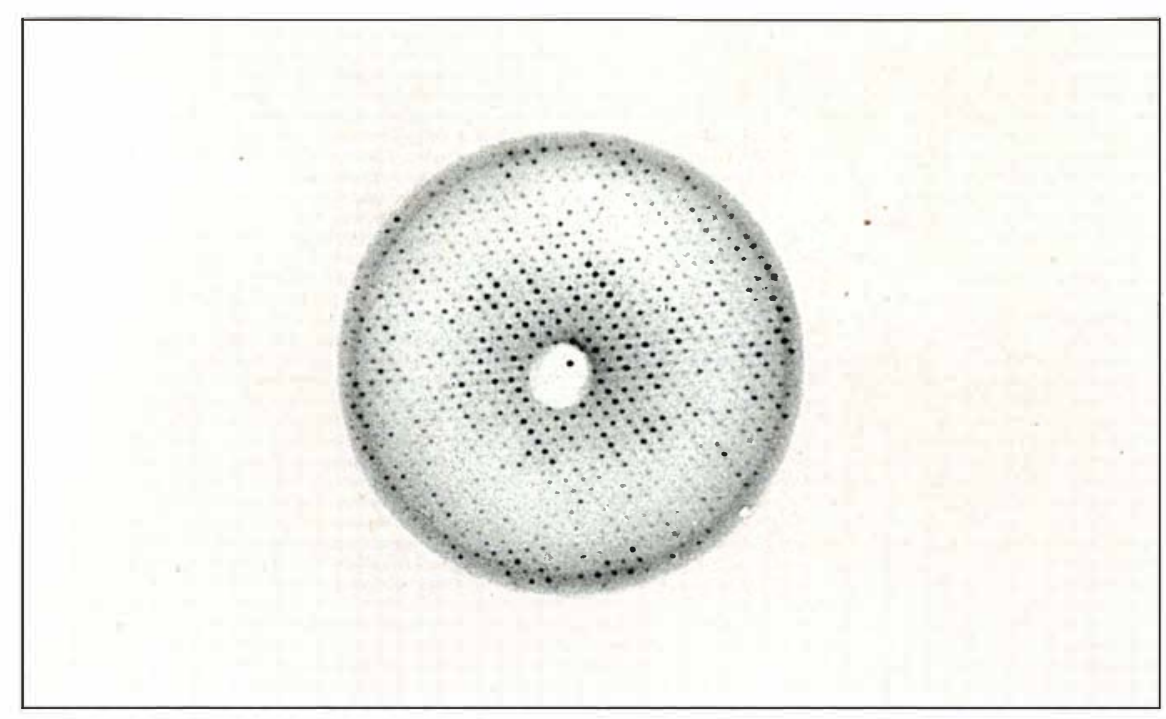

Figure 3a. Image de diffraction obtenue avec un monocristal de sérylARNt synthétase. La position des taches est déterminée par les paramètres de la maille cristalline (dans ce cas : $12,81 \times 9,07 \times 6,98 \mathrm{~nm}^{3}$ ) et leur intensité par la structure de la molécule. Dans le type de collection utilisé (cliché de précession), les mouvements de l'échantillon et du film sont couplés de telle façon que les taches soient disposées sur un réseau périodique dont les paramètres sont inversement proportionnels à ceux du réseau cristallin. (Cliché de Nicolas Nassar, EMBL Grenoble.)

temps de prise de l'image (une fraction de seconde). En revanche, les échantillons peuvent rester dans un faisceau de rayons $\mathrm{X}$ de laboratoire pendant plusieurs heures.

L'impossibilité d'observer une molécule unique avec les rayons $\mathrm{X}$ impose d'utiliser un échantillon contenant un grand nombre des molécules. La seule façon de le faire, sans perdre la résolution, est d'utiliser un monocristal. Notons qu'en microscopie électronique, avec les plus faibles doses (100 électrons par $\mathrm{nm}^{2}$ ), on a le même problème et que, là aussi, on utilise alors un monocristal. La périodicité de l'organisation des molécules a comme conséquence que les ondes diffusées par chacune des molécules contribuent de façon cohérente au signal. Ce signal est ce que l'on appelle un image de diffraction; elle est constituée de taches plus ou moins intenses (figure $3 a$ ). La position de ces taches ne dépend que de la périodicité du réseau cristallin formé par les molécules et elle ne contient donc pas d'informations sur la structure de la molécule. Cette informa- tion est contenue dans les intensités des taches. Elle est par elle-même insuffisante; pour reconstituer une image de la molécule à partir de l'image de diffraction, il faut connaître les phases relatives des ondes qui ont contribué à former chacune des taches. Dans le cas du microscope, l'image est reconstituée par des lentilles qui recombinent les ondes diffusées avec leurs phases relatives. Avec les rayons $\mathrm{X}$, cette information sur les phases est obtenue, le plus souvent, en collectant des images de diffraction à partir de cristaux de la même molécule sur laquelle est venu se fixer, en un tout petit nombre de sites, un atome lourd (plomb, or, uranium par exemple : les cystéines jouent souvent un rôle dans cette fixation). L'ensemble des données collectées sur le cristal natif et sur plusieurs types de cristaux contenant des atomes lourds permet, si les données sont de bonne qualité, de déterminer, par le calcul, la structure de la molécule.

Quelques chiffres : on utilise des cris $\mathrm{m} / \mathrm{s} n^{\circ} 4$, vol. 7, auril 91 


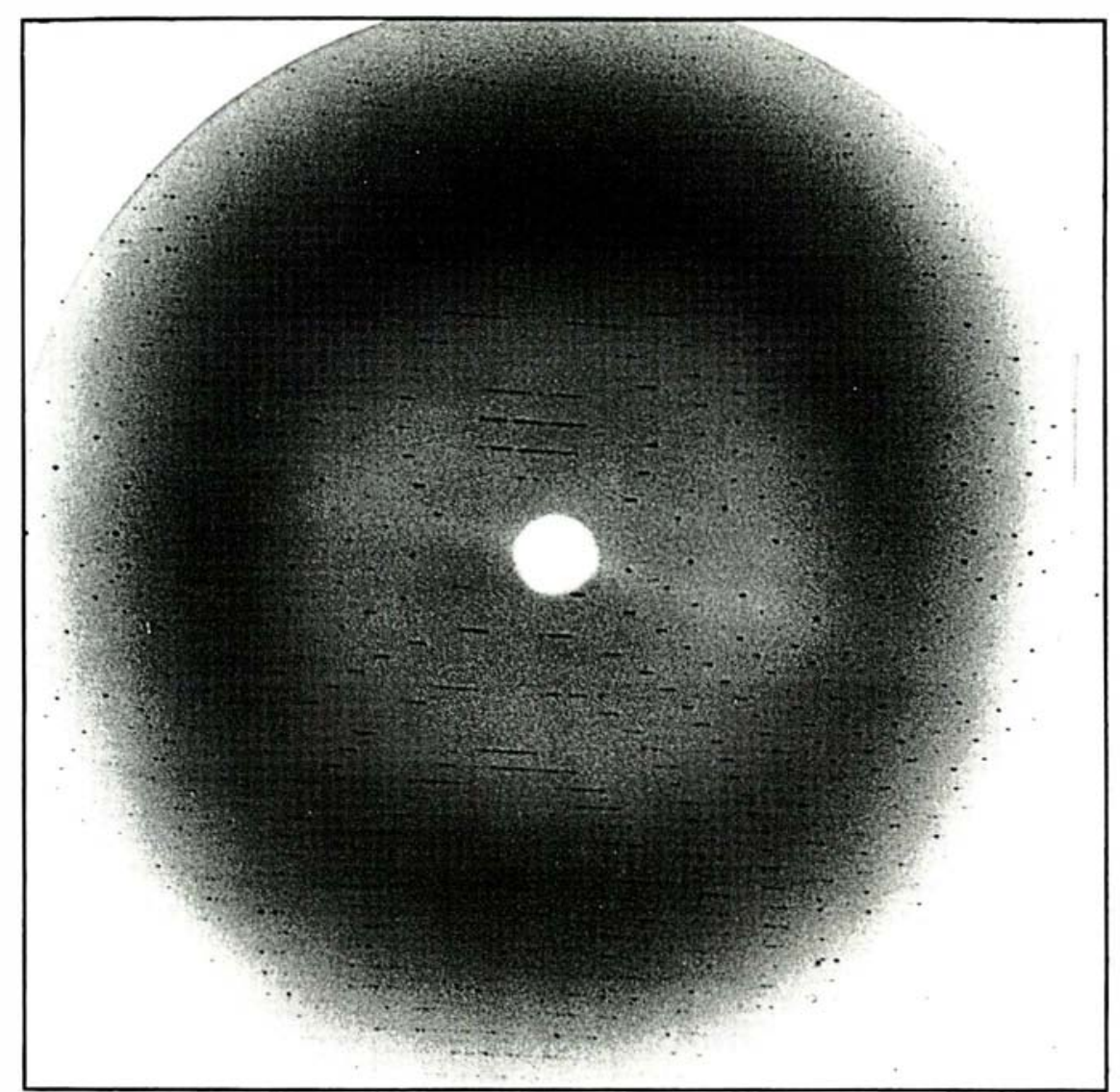

Figure 3b. Image de diffraction obtenue avec un film fixe et une très légère rotation du cristal. C'est ce type d'image qui est utilisé pour collecter les données permettant de résoudre la structure. Ici l'échantillon est un cristal de neuraminidase du virus $B$ de la grippe. Le cliché a été pris auprès de la source de rayonnement synchrotron d'Orsay (LURE). Pour résoudre la structure, il faut prendre plusieurs centaines de tels clichés et ensuite mesurer l'intensité de toutes les taches à l'aide d'un densitomètre. Cela est une tâche très fastidieuse et l'on tend de plus en plus à collecter les données avec un détecteur électronique ou des plaques photosensibles qui permettent de simplifier la mesure de ces intensités. (Cliché de Wim Burmeister, EMBL Grenoble.)

taux qui ont un volume compris entre $10^{-3}$ et $1 \mathrm{~mm}^{3}$ (ce qui correspond à un nombre de $10^{10}$ à $10^{15}$ molécules). Il faut, avec un équipement de laboratoire, environ 1 heure pour collecter les données d'une image de diffraction. Cette image fut pendant longtemps enregistrée sur un film photographique. On utilise maintenant des détecteurs électroniques qui permettent d'éviter le fastidieux travail de lecture des films. La technique la plus récente utilise des $\mathrm{m} / \mathrm{s} n^{\circ} 4, \mathrm{vol} .7$, auril 91 centaines d'images de diffraction. Cela demande plusieurs mois (ou années) et de nombreux cristaux car, après quelques heures d'irradiation aux rayons $\mathrm{X}$, les cristaux commencent à se détériorer. Le nombre de données à collecter croît avec la résolution recherchée et la taille de la molécule.

De nouvelles sources de rayons $\mathrm{X}$, beaucoup plus intenses que les sources traditionnelles de laboratoire, sont disponibles depuis quelques années. Elles utilisent le rayonnement émis par des électrons tournant à très grande vitesse dans un anneau circulaire (rayonnement synchrotron). Le rayonnement $\mathrm{X}$ émis est polychromatique; le domaine de longueur d'onde dépend des caractéristiques de l'anneau. Une telle source est en fonctionnement à Orsay depuis plus de dix ans (figure $3 b$ ). Les rayons $\mathrm{X}$ émis ont des longueurs d'onde comprises entre 0,1 et $0,2 \mathrm{~nm}$. A l'aide d'un mono-chromateur, on peut prélever un faisceau de longueur d'onde bien définie et envoyer sur l'échantillon entre $10^{-1}$ et $10^{-2}$ photon monochromatique par $\mathrm{nm}^{2}$ et par seconde. Un ensemble de données peut être collecté en quelques jours. Cela est un progrès certain qui accélère considérablement la détermination des structures. Cela permet aussi d'améliorer la qualité des données et par conséquent la précision de la détermination. Pour trouver une structure, il faut avoir mesuré un nombre suffisant de taches avec une précision statistique satisfaisante. Pour cela il faut que le cristal ait reçu un nombre suffisant de photons. $\mathrm{Ce}$ nombre est le même avec une source traditionnelle et avec le rayonnement synchrotron, mais, dans ce dernier cas, il faut 100 à 10000 fois moins de temps. L'expérience prouve qu'alors l'effet destructeur du rayonnement est quelque peu réduit. C'est une des raisons pour lesquelles les données collectées sont, dans ce cas, meilleures et la structure déterminée avec plus de précision. Il en est d'autres : par exemple, on peut utiliser des rayons $\mathrm{X}$ de courte longueur d'onde $(0,1 \mathrm{~nm}$ ou moins), dont les effets destructeurs sont de moindre importance.

Le goulot d'étranglement dans la cristallographie des protéines est 


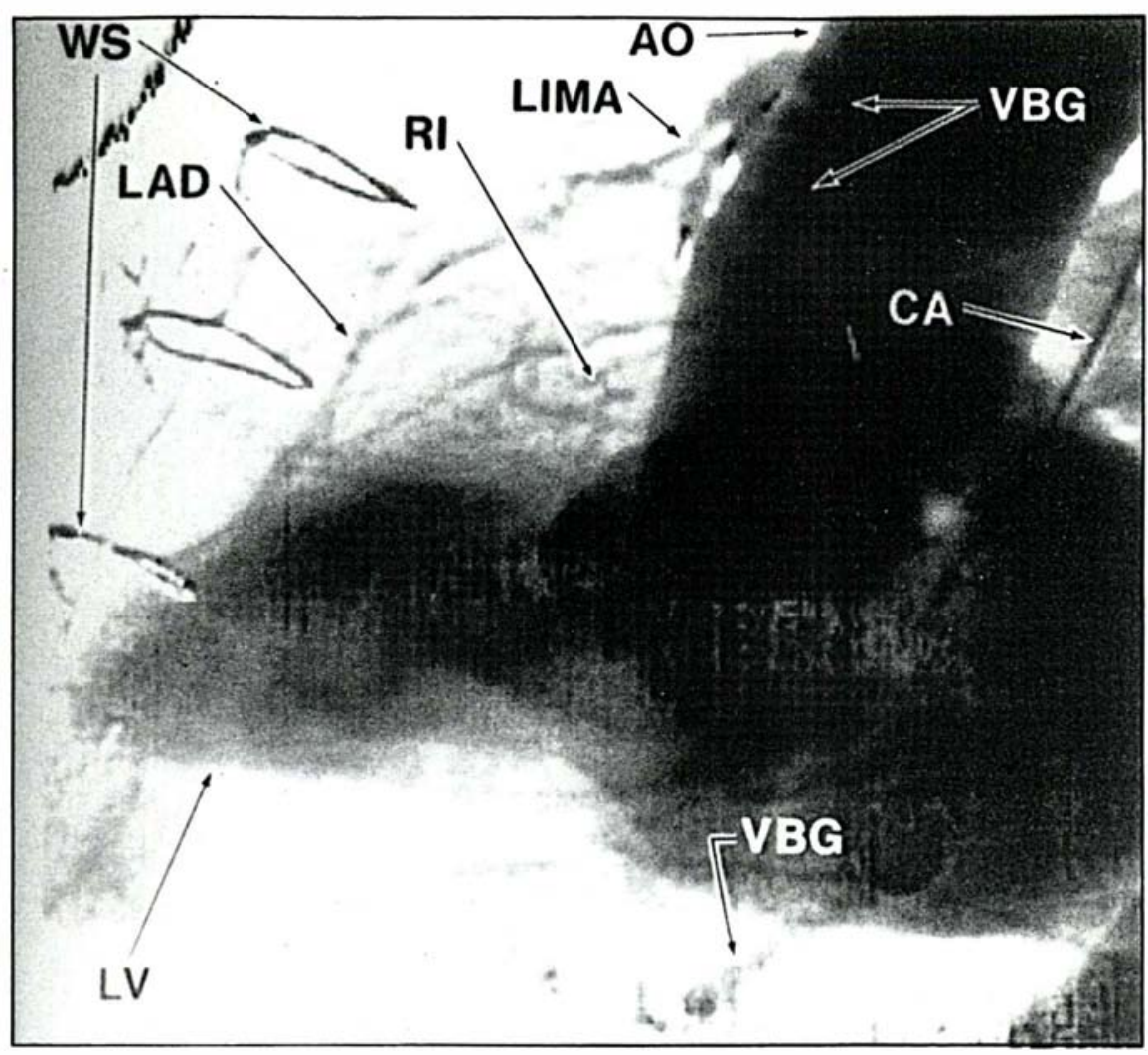

\section{RÉFÉRENCES}

8. Wüthrich K. Protein structure determination in solution by nuclear magnetic resonance spectroscopy. Science $1989 ; 243$ : 45-50.

9. Hendrickson WA, Smith JL, Sheriff S. Direct phase determination based on anomalous scattering. In : Wyckoff HW, et al., eds. Methods in Enzymology 115 part B. London : Academic Press, 1985: 41-55.

10. Moffat K. Method in MADness. Nature $1988 ; 336$ : 422-3.

11. Moffat K. Time resolved macromolecular crystallography. Ann Rev Biophys Biophys Chem 1989; 18 : 309-32.

12. Hajdu J, Johnson LN. Progress with Laue diffraction studies on protein and virus crystals. Biochemistry $1990 ; 29$ : 1669-78.

13. Schlichting I, Almo SC, Rapp G, el al. Time resolved X-ray crystallographic study of the conformational change in Ha-Ras p21 protein on GTP hydrolysis. Nature 1990 ; $345: 309-15$.

14. Gruner SM. Time resolved X-ray diffraction of biological materials. Science 1987 ; 238: 305-12.

15. Rubenstein E, Giacomini JC, Gordon $\mathrm{HJ}$, el al. Synchrotron radiation coronary angiography with a dual-bearn, dualdetector imaging system. Nucl Instr Meth

Figure 4. Angiogramme obtenu à I'aide des rayons $X$ du rayonnement produit au Stanford Synchrotron Radiation Laboratory, USA. Le sujet était un homme de 62 ans chez lequel avait été fait un pontage quatre ans avant. L'agent de contraste (Renografin-76) a été injecté dans la veine cave supérieure. Une obstruction de l'artère antérieure gauche (LAD) est bien visible. $D^{\prime}$ après Rubinstein et al., 1990, ce travail a été financé par le National Institut of Health et le Department of Energy.) $A O$ : aorta, aorte ; LIMA : left internal mammary bypass graft, pontage veineux; $R I$ : ramus intermedius artery, rameau de l'artère intermédiaire; $L A D$ : left anterior descending artery, artère descendante antérieure gauche; $L V$ : left ventricle, ventricule gauche; $C A$ : cathéter; $C L$ : surgical clips, clips chirurgicaux; WS : wire sutures, sutures métalliques.

l'obtention de cristaux de qualité et de taille suffisantes. De considérables efforts sont faits pour comprendre quels sont les facteurs qui déterminent la croissance cristalline. Des congrès entiers sont dédiés à ce problème. Mais, pour le moment, il n'est pas encore maîtrisé et l'obtention de cristaux relève encore d'un empirisme mitigé d'expérience et d'intuition. Dans de nombreux cas, on ne parvient à obtenir que des microcristaux ayant des dimensions de l'ordre de $10^{-3} \mathrm{~nm}$. Le nombre de molécules est alors trop faible et il n'est pas possible de collecter des données, même avec le rayonnement synchrotron produit par une source telle celle d'Orsay; une source plus intense est alors nécessaire pour essayer d'utiliser de tels cristaux.

Au début, le rayonnement synchrotron était utilisé sur des machines construites par et pour les physiciens nucléaires qui utilisaient le faisceau d'électrons. Ces machines n'étaient donc pas optimalisées pour la production des rayons X. Des sources sont maintenant spécialement construites pour fournir des faisceaux très intenses de rayons X. L'une d'elles, réalisée à l'échelle européenne, sera 
en fonctionnement à Grenoble en 1993. Dénommée European Synchrotron Radiation Facility (ESRF), elle est prévue pour être la source la plus intense de ray'ons $\mathrm{X}$ au monde. Ses faisceaux les plus intenses sont prévus pour permettre d'utiliser, au niveau de l'échantillon, de 100 à 1000 fois plus de photons (c'est-à-dire de 1 à 10 photons par $\mathrm{nm}^{2}$ ) que dans la source d'Orsay.

De telles sources sont nécessairement très coûteuses (de l'ordre de 2 milliards de francs). Elles ne sont évidemment pas spécialement construites pour les biologistes et les médecins, mais plutôt pour les physiciens. Cependant, elles sont d'une grande utilité pour les biologistes qui étudient les macromolécules.

La première utilité d'une source telle que celle de l'ESRF est, comme nous l'avons dit, de réduire le temps nécessaire au recueil des données et de permettre de travailler avec des microcristaux, étendant ainsi le champ d'application de la détermination des structures par rayons X. Mais ce n'est absolument pas la seule. J'ai évoqué le problème du calcul de l'image lié à la perte d'information sur les phases des ondes diffractées. La solution traditionnelle à ce problème, à savoir l'utilisation de dérivés d'atomes lourds, n'est pas parfaite. La recherche de ces dérivés est longue, difficile et fastidieuse. Le rayonnement synchrotron, parce qu'il n'est pas monochromatique, autorise une autre approche. Il est alors possible, en réglant le monochromateur, de collecter des images de diffraction avec plusieurs longueurs d'onde. Or il se trouve que, pour certains éléments, il y a de considérables variations du pouvoir diffusant avec la longueur d'onde des rayons X. En mesurant la diffraction à plusieurs longueurs d'onde, on peut donc changer la contribution de certains atomes de la molécule et disposer ainsi d'une information équivalente à celle que donnent les mesures faites sur des molécules comportant des dérivés d'atomes lourds $[9,10]$. L'un des éléments qui permet ce type de détermination est le sélénium. Or cet élément peut être introduit à la place du soufre dans une protéine produite par génie génétique, en faisant croître les bactéries en présence de sélénométhionine. La méthode peut donc être d'application assez générale et plusieurs travaux l'utilisant ont été présentés à des congrès au cours de l'été 1990.

La connaissance de la structure d'une protéine n'est pas une fin en soi, mais elle est nécessaire pour comprendre comment fonctionne une enzyme donnée. Idéalement, il serait très instructif de pouvoir suivre les modifications de structure d'une enzyme au cours de la réaction qu'elle catalyse. Pour cela, il faut être capable de collecter les données en un temps qui soit plus court que les temps de vie des divers états intermédiaires qui apparaissent au cours de la réaction. Avec la méthodologie décrite plus haut, qui utilise un rayonnement monochromatique, il faut, même avec le rayonnement synchrotron, plusieurs heures pour avoir un ensemble de données permettant la détermination d'une structure. Un gain de temps considérable peut être obtenu si l'on utilise un rayonnement polychromatique (ou blanc). Dans ces conditions, il n'est plus nécessaire de multiplier les mesures prises sous différentes orientations du cristal et un ensemble de données utiles peut être obtenu à partir d'un seul cliché. Cette méthode, dite méthode de Laue, est connue depuis les débuts de la cristallographie ; on a démontré qu'elle était applicable aux macromolécules $[11,12]$ et que des expériences permettant de suivre un phénomène en fonction du temps étaient alors possibles. Il faut alors, avec les sources de rayonnement synchrotron existantes, environ 1 seconde pour avoir des données utiles sur une protéine et 30 secondes sur un petit virus.

Le travail le plus intéressant publié à ce jour [13] est sans doute celui qui porte sur les changements de conformation du produit de l'oncogène Ras au cours de l'hydrolyse du GTP. Il s'agit d'un phénomène assez lent (demi-vie de l'ordre de 40 minutes), donc relativement facile à étudier. $\mathrm{Au}$ départ, le cristal contient du GTP sous une forme inactive. Cette structure est étudiée par les méthodes traditionnelles. Un éclair lumineux active le GTP et la structure est alors déterminée, par la méthode de Laue, 4 minutes et 14 minutes après cette activation, avec des temps de pose de 45 secondes. Après une journée, l'état final est étudié par la méthode classique. Le but de ce travail était de voir comment les mutations qui activent l'oncogène peuvent modifier le mécanisme d'hydrolyse du GTP.

Un des buts des sources très intenses, comme celle de l'ESRF, est de pouvoir faire de telles études sur des mécanismes beaucoup plus rapides à l'échelle de la milliseconde et peut-être même de la microseconde, voire de la picoseconde. Pour essayer d'atteindre cet objectif, il faut être capable de déclencher la réaction sur toutes les molécules du cristal au même instant. Il faut ensuite modifier le fonctionnement du synchrotron pour qu'il produise les rayons $\mathrm{X}$ pendant une seule impulsion, très courte mais très intense. Il faut enfin disposer d'un détecteur qui soit suffisamment rapide pour suivre la réaction. Ce sont là des défis majeurs, qui exigent la collaboration de biologistes, de physiciens et de chimistes de premier plan. Mais c'est un défi qui vaut la peine d'être relevé.

En attendant, certaines expériences sont plus faciles à réaliser parce que l'on ne cherche pas à obtenir une information structurale à l'échelle atomique et que l'on a donc besoin d'un beaucoup plus petit nombre de données. C'est, par exemple, le cas de la contraction musculaire, ou de mécanismes d'auto-assemblage d'un virus ou de microtubules. Ces approches ouvrent donc un champ vaste et prometteur [14].

\section{Imagerie médicale}

L'angiographie, malgré les progrès considérables permis par l'angiographie numérique, est un problème typique d'imagerie médicale reposant sur les rayons $\mathrm{X}$, qui n'a pas encore été résolu de manière satisfaisante. La méthode traditionnelle utilise l'injection de produits iodés par voie artérielle et ne peut donc pas être employée pour des examens de routine. Plus récemment, différentes méthodes ont été mises au point qui permettent l'injection des produits iodés par voie veineuse. Elles utilisent deux images dans lesquelles la contribution relative du sang (marqué à l'iode) est différente, par exemple l'une étant prise avant et l'autre après l'injection de l'iode. Cela pose 
un problème pratique d'immobilité du patient. Une façon plus efficace d'obtenir ces deux images est de les prendre en utilisant des rayons $\mathrm{X}$ de part et d'autre du seuil d'absorption de l'iode $(33,16 \mathrm{Kev})$. Dans ces conditions, la contribution du sang iodé à l'absorption des rayons $\mathrm{X}$ est très différente pour les deux images et une soustraction de ces images permet une visualisation très claire du sang. Cela est très facile à réaliser avec une source de rayonnement synchrotron qui émet un rayonnement polychromatique [15]. Un dispositif permet d'envoyer alternativement, ou même simultanément, deux faisceaux dont les longueurs d'onde sont de part et d'autre du seuil d'absorption. Plusieurs groupes travaillent à la mise au point d'une méthode qui, si elle tient ses promesses, conduira peut-être à la construction de petites sources de rayonnement synchrotron qui seraient installées dans les hôpitaux. Une source d'intensité maximale est cependant nécessaire si l'on veut collecter les clichés assez rapidement pour obtenir une image dynamique de la circulation. L'avenir dira si la mise en œuvre de moyens aussi lourds apportera quelque chose d'utile par rapport aux progrès que l'on peut attendre de méthodes plus légères comme l'imagerie RMN.

\section{Conclusions}

Les quelques exemples discutés dans cet article des progrès récents dans l'exploration de la matière vivante et de l'homme par des méthodes physiques contribueront peut-être à rappeler aux lecteurs de médecine/sciences que l'avancée des connaissances est toujours multidisciplinaire.

L'imagerie médicale est une évidente et éclatante conquête de ces approches. A l'heure où la recherche biologique tend à s'éloigner de son commerce exclusif avec le gène pour s'intéresser de plus en plus aux structures et aux formes, nul doute que les physiciens et leurs appareillages soient ainsi appelés à occuper une place croissante dans le concert des décrypteurs du vivant

\section{TIRÉS A PART}

\section{Summary}

Some new plyesical methods in biology and medicine

Recent developments in physical instrumentation have provided biologists with new tools. NMR is now a widely used method to determine macromolecular structures : initially limited to low molecular weight proteins, recent improvements, such as 3-D and 4-D NMR coupled with isotopic labelling, make the method useful for the analysis of higher molecular weight proteins (30 000 and possibly 40000 ). Tunneling microscope may become a tool to visualise molecules at very high resolution and follow macromolecular assemblies. New X-ray sources, as provided by synchrotron radiations, open new prospects in structure determinations; one of them is the possibility of following structural modifications during a reaction. These intense $\mathrm{X}$-ray sources may also have applications in imaging for medical applications. 